\title{
Rapid identification and characterization of hammerhead-ribozyme inhibitors using fluorescence-based technology
}

\author{
Andreas J enne ${ }^{1,2}$, J örg S. Hartig ${ }^{1}$, Nicolas Piganeau ${ }^{1,3}$, Andreas Tauer ${ }^{4}$, Dmitry A. Samarsky5, \\ Michael R. Green ${ }^{5}$, J ulian Davies ${ }^{6}$, and Michael Famulok ${ }^{1 *}$
}

\begin{abstract}
${ }^{1}$ Kekulé-Institut für Organische Chemie und Biochemie, Gerhard-D omagk-Str. 1, 35121 Bonn, Germany. ${ }^{2}$ Current address: NascaCell GmbH , Eisvogelweg 2, D-83209 Prien am Chiemsee, Germany. ${ }^{3}$ Current address: Aventis Gencell, 3825 Bay Center Place, Hayward, CA 94545, U SA. 4 Institut für Biochemie, LM U M ünchen, Feodor-Lynen-Str. 25, 81377 M ünchen, Germany. ${ }^{5} \mathrm{H}$ oward Hughes M edical Institute, Programs in GeneFunction and Expression and M olecular M edicine, University of M assachusetts M edical School, 373 Plantation St., Worcester, M A 01605, USA. ${ }^{6}$ D epartment of M icrobiology and Immunology, University of British Columbia, Vancouver, BC V6T 1Z3, Canada. *Corresponding author (m.famulok@uni-bonn.de).
\end{abstract}

\begin{abstract}
The ability to rapidly identify small molecules that interact with RNA would have significant clinical and research applications. Low-molecular-weight molecules that bind to RNA have the potential to be used as drugs. Therefore, technologies facilitating the rapid and reliable identification of such activities become increasingly important. We have applied a fluorescence-based assay to screen for modulators of hammerhead ribozyme (HHR) catalysis from a small library of antibiotic compounds. Several unknown potent inhibitors of the hammerhead cleavage reaction were identified and further characterized. Tuberactinomycin A, for which positive cooperativity of inhibition in vitro was found, also reduced ribozyme cleavage in vivo. The assay is applicable to the screening of mixtures of compounds, as inhibitory activities were detected within a collection of 2,000 extracts from different actinomycete strains. This approach allows the rapid, reliable, and convenient identification and characterization of ribozyme modulators leading to insights difficult to obtain by classical methodology.
\end{abstract}

Keywords: antibiotic libraries, combinatorial libraries, hammerhead ribozyme, high-throughput screening, RNA-drug interaction

Much like proteins, RNA molecules can fold into complex threedimensional structures, allowing them to form surfaces and binding pockets to specifically interact with other molecules. RNA performs highly precise functions in a number of essential cellular processes, such as protein synthesis, transfer RNA (tRNA) maturation, and messenger RNA (mRNA) splicing. Furthermore, it has been found that certain structural elements in the mRNA of the human immunodeficiency virus (HIV), namely the trans-activating region (TAR) and the Rev response element ( $R R E$ ), are critical for retroviral replication ${ }^{1}$. These findings, together with the inability thus far to identify any natural RNA repair mechanisms, make functional RNA molecules attractivedrug targets in pharmaceutical research programs ${ }^{2-4}$. Indeed, antibiotics targeting distinct sites in ribosomal RNA ( $r R N A$ ) have been known for a long time and are used in clinical practice to fight bacterial infections. For example, aminoglycosides of the 2-deoxystreptamine family specifically interfere with prokaryotic translation by binding to the decoding site of the 16S rRNA (ref. 5) or inhibit virus production by interacting with the RRE and TAR sequences in HIV ${ }^{6-8}$. Furthermore, small-molecule drugs can influence the cleavage activity of natural ribozymes-15. It has been suggested that the H HR could be used as a model system for the analysis of RNA/small-molecule interactions, in that it belongs to the most exhaustively characterized catalytic RNAs (refs 2, 4, 14, 16-19).

Besides rRNA (refs 5, 20), there have been efforts to target catalytic RNAs in viruse ${ }^{21}$ and pathogenic microorganisms with lowmolecular-weight molecules 2,17 . This seems particularly attractive in that autocatalytic splicing processes and ribozymatic activities involved in virus replication are species-specific, play key roles in the life cycle of microorganisms or viruses, and do not occur in higher eukaryotic cells. For that reason, catalytic RNA is increasingly being considered as a promising drug target 2,17 . On the other hand, the ability to identify compounds that modulate RNA activity in highthroughput screens is important not only to target biologically active RNA molecules, but also to be able to overcome the growing problem of the global dissemination of bacterial resistance to many of the current antibiotics $22-24$. These considerations prompted us to design approaches enabling the rapid and convenient identification and characterization of new inhibitors of the H HR, and, ultimately, other functionally interesting RNA molecules.

Recently, we have described an assay that enables the rapid kinetic analysis of HHRs by fluorescence resonance energy transfer (FRET) ${ }^{25}$. In contrast to conventional methods, the assay is eminently suited for the high-throughput analysis of ribozyme cleavage activities because it allows the nonradioactive and automated characterization of many RNA-catalyzed reactions in microtiter plates (Fig. 1A,B). Here we report the rapid identification and characterization of new HHR inhibitors from two small compound libraries using this approach. One library was a collection of antibiotics from which a modulatory effect on RNA molecules could be assumed on the basis of previous data; the other was a collection of extracts from 2,000 different actinomycete strains.

\section{Results and discussion}

Inhibitor screening. We have applied a fluorescence-based assay to test 96 antibiotics as well as 2,000 bacterial extracts from actino- 
A

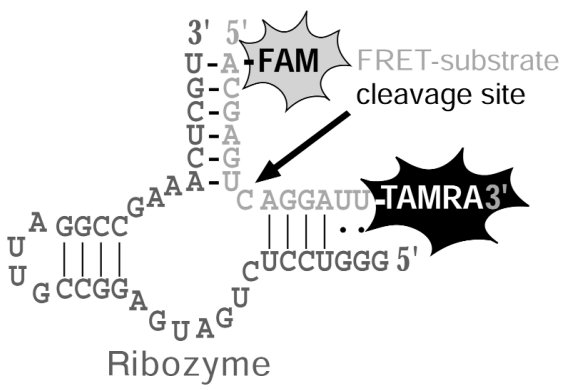

D

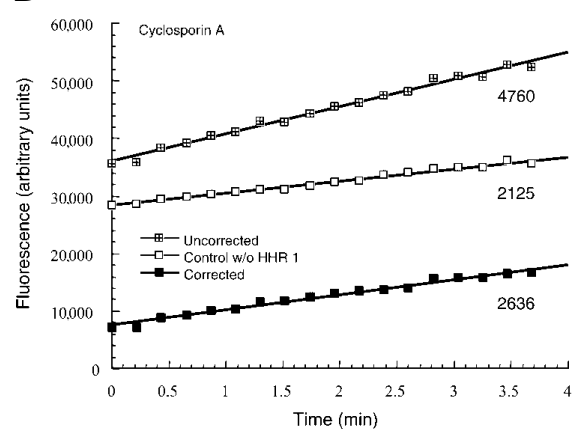

B

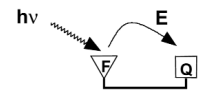
Substrate binding (1)

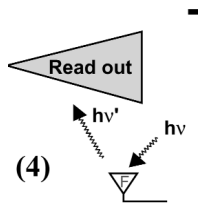

回

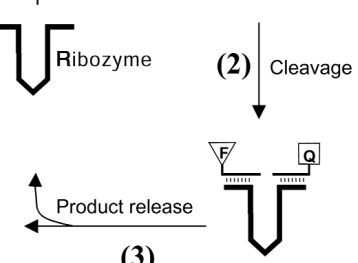

(3)
C

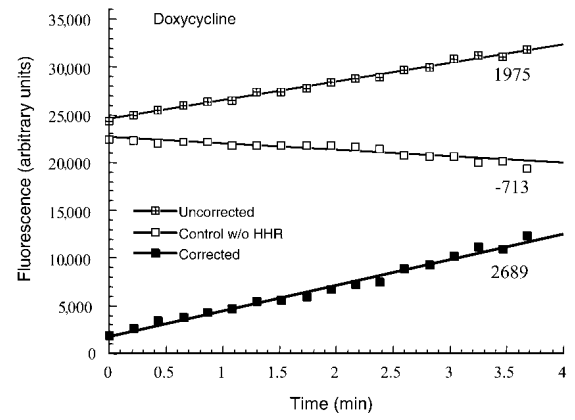

Figure 1. Principle of the assay and primary fluorescence measurements with HHR1/SL1. (A) Sequences of HHR 1 and its substrate. (B) Principle of the FRET assay. (1) Binding of the FRET substrate to the ribozyme results in the formation of the catalytic complex. In the uncleaved substrate the fluorescence signal of the fluorophore (F) is intramolecularly quenched by the adjacent quencher dye (Q). (2) Cleavage of the FRET substrate by the hammerhead ribozyme. (3) Dissociation of the reaction products from the ribozyme. Neutralization of the FRET effect results in an increase in fluorescence $\left(h v^{\prime}\right)$. The ribozyme is available for the next turnover. (4) The increasing fluorescence signal reflecting the cleavage rate under multiple-turnover conditions is monitored as a measure for ribozyme activity. (C) Time-dependent increase of the fluorescence signal for the reaction in the presence of doxycycline resulted in a decrease of the fluorescence signal in the control sample (slope: $-713 \mathrm{Fu} / \mathrm{min}$ ). (D) The reaction in the presence of cyclosporin A. Here, fluorescence increase was observed not only in the reaction containing the ribozyme (slope: 4,760 Fu/min), but also in the control sample (slope: $2,125 \mathrm{Fu} / \mathrm{min}$ ). When subtracted from the nonspecific fluorescence, the initial reaction rates appeared to be much lower in the presence of cyclosporin A (corrected slope: $2,636 \mathrm{Fu} / \mathrm{min}$ ). Data points and linear fit for the uncorrected reaction, the corresponding control reaction without HHRI and the corrected values are shown (see Experimental Protocol). The slopes of the lines corresponding to the initial reaction rates are also given ( $\mathrm{Fu} / \mathrm{min})$.

mycetes with potential antibiotic activity for their ability to modulate the cleavage activity of an in-trans hammerhead ribozyme H HR1 (Fig. 1A). The kinetic parameters of HHR 1 were determined previously under similar conditions under which the screening was performed ${ }^{25}$. In the assay, a fluorophore-labeled oligonucleotide ("FRET-oligonucleotide") serves as a substrate for the hammerhead ribozyme. Upon cleavage of the FRET-substrate, a fluorescence signal proportional to the cleavage activity of HHR1 is generated. By fluorescence monitoring in the linear phase of the reaction, the initial ribozyme-catalyzed cleavage rate could befollowed and quantified in subsequent analyses (Fig. 1B). Recently, the principal applicability of our assay for the kinetic characterization of RNA cleavage reactions was confirmed for other HHRs (ref. 26).

The inhibitor screening was performed in 96-well plates using a fluorescence kinetic reader. The prototype of this assay, that is, automated measurements with manual pipetting, can be applied to record and analyze at least 480 different hammerhead reactions per day. All reactions were performed under multiple-turnover conditions at subsaturating ribozyme concentrations and with substrate in excess. Hammerhead cleavage was initiated by simultaneously adding $\mathrm{M} \mathrm{gCl}_{2}$ and the sample to be analyzed to a preincubated mixture of the ribozymeand theFRET substrate. Kinetics were measured in the initial linear phase of the reaction to ensure that the substrate concentration was not rate limiting. In principle, inhibition was detected by the lower increase of the fluorescence signal relative to the noninhibited $\mathrm{HHR}$ reaction.

To improve the accuracy of our assay, a control sample containing no HHR was measured on the same plate for each cleavage reaction ( control sample = FRET substrate, compound to betested, no HHR). The fluorescence signal of the control remained unchanged during the time of the measurement (data not shown), although in some rare cases an increase or decrease of the control fluorescence was observed over time (Fig. 1C,D). Here, subtraction of the fluorescence signal in the control allowed correction for possible effects of the compound on the FRET substrate that do not result from ribozyme activity, such as nonspecific fluorescence quenching or enhancement, RNA multimerization, or RNase degradation. For example, in the presence of doxycycline and cyclosporin A (Fig. 1C,D), cleavage is inhibited as indicated by the smaller corrected values (fluorescence units, Fu/min =2,689 and 2,636, compared to the corrected slope of the uninhibited reaction. Thus, correction led to significantly lower errors in the determination of initial reaction velocities.

We then screened a library of 96 known antibiotics

Table 1. Members of the 96 compounds screen with inhibitory activity

\begin{tabular}{|c|c|c|c|c|}
\hline \multirow[t]{2}{*}{ Entry ${ }^{a}$} & \multirow[t]{2}{*}{ Compound (MW) } & \multicolumn{2}{|c|}{ Relative activity ${ }^{b}$} & \multirow[t]{2}{*}{$\mathbf{K}_{\mathbf{i}}(\mu \mathbf{M})$} \\
\hline & & FRET & $55^{\prime}-32 \mathrm{P}$ & \\
\hline 2 & Adriamycin RDF (543.5) & 0.12 & 0.13 & 14 \\
\hline 8 & Bisbenzimide (629) & 0.00 & 0.08 & 3 \\
\hline 15 & Chelocardin (441; DMSO) & 0.15 & 0.20 & \\
\hline 27 & Diminazene aceturate (515.5) & 0.00 & 0.12 & 20 \\
\hline 31 & 5-epi-Sisomicin (447.6) & 0.00 & 0.03 & 1 \\
\hline 38 & Gentamicin C complex (700) & 0.22 & & 10 \\
\hline 39 & Gentamicin C1A sulfate (700) & 0.39 & & 32 \\
\hline 40 & Gentamicin sulfate (709) & 0.69 & & 73 \\
\hline 41 & Gramicidin S. $\mathrm{HCl}(1214.5)$ & 0.03 & 0.06 & 23 \\
\hline 53 & Neamine (322.4) & 0.61 & & 90 \\
\hline 54 & Neomycin B (614) & 0.33 & & 38 \\
\hline 55 & Neomycin sulfate (908.9) & 0.16 & & 18 \\
\hline 62 & Paramomycin (615.7) & 0.63 & & 65 \\
\hline 84 & Tetracycline (444.1) & 0.61 & & \\
\hline 91 & Tuberactinomycin A (701) & 0.01 & 0.00 & 1 \\
\hline 92 & Tuberactinomycin B (685) & 0.03 & 0.01 & 0.1 \\
\hline
\end{tabular}

aEntry numbers refer to the numbering of all 96 compounds tested. The complete set of the 96 compound screen is available as Supplementary Table 1 in the Web Extras page of Nature Biotechnology Online.

${ }^{b}$ Fraction of activity at $100 \mu \mathrm{M}$ of antibiotic in relation to the noninhibited reaction. For antibiotics with a strong inhibitory effect, $\mathrm{K}_{i}$ values are also given. Measurements were carried out in triplicate. Chelocardin was tested at concentrations below $100 \mu \mathrm{M}$, because solubility prevented preparation of a $1 \mathrm{mM}$ stock solution. 
A

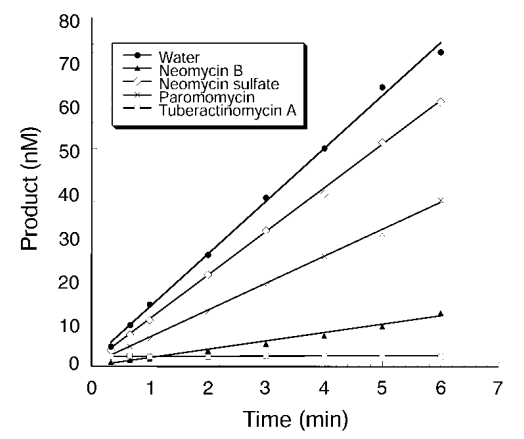

B

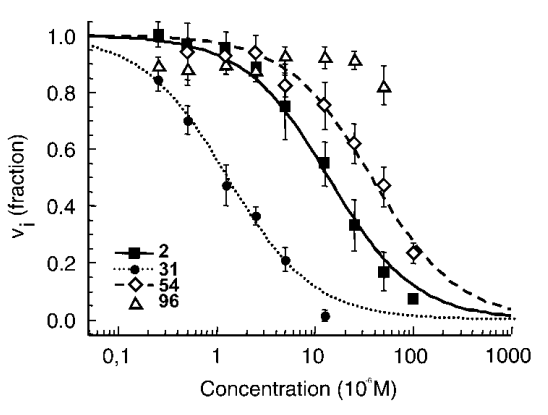

C

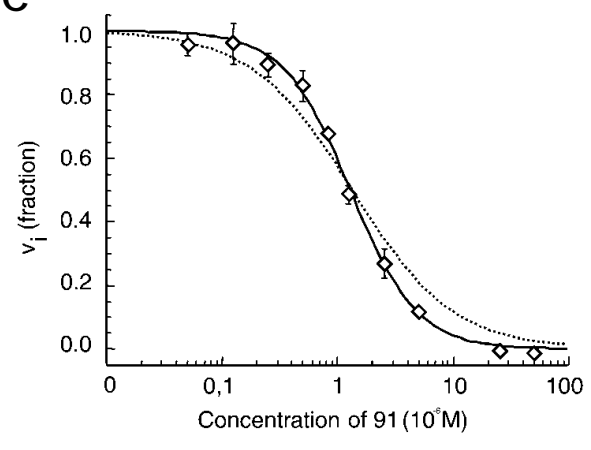

Figure 2. ${ }^{32} \mathrm{P}$-Labeling experiments under single- and multiple-turnover conditions, $\mathrm{K}_{\mathrm{i}}$ determinations and cooperativity. (A) Time course of HHR1 shown representatively for neomycin B, neomycin sulfate, paromomycin, TubA. No cleavage was detected in the presence of $100 \mu M$ TubA. (B) Influence of compounds 2 (Adriamycin; straight line, $\left.K_{i}=13.7 \mu \mathrm{M}\right), 31$ (5-epi-sisomicin; dotted line), 54 (neomycin B; dashed line), and 96 (viomycin; triangles) on HHR 1 cleavage. $v_{i}$, Initial reaction velocity. (C) Inhibition by TubA. Dotted line: no cooperativity, $K_{i}=1.3 \pm 0.2 \mu M$. Straight line: cooperativity factor $1.5, K_{i}=1.3 \pm 0.1 \mu \mathrm{M}$. In a single measurement $K_{i}$ values could be obtained in triplicate.

(Supplementary Table 1) that was limited to molecules from which an effect on RNA might be expected on the basis of a large set of previous data10,12,15,21. (See Supplementary Table 1 in the Web Extras page of Nature Biotechnology Online.) Indeed, 16 compounds showed an inhibitory effect compared to the uninhibited reaction (relative activity $<0.7$ ), and among them eight novel inhibitors of the HHR-cleavage reaction were identified: Adriamycin RDF (doxorubicin), bisbenzimide, chelocardin, diminazene aceturate, gramicidin S, 5-epi-sisomicin, tuberactinomycin A (TubA), and tuberactinomycin B (TubB) (Table 1). In Table 1 and in Supplementary Table 1 the corrected initial velocities for all compounds are listed as relative values of activity with respect to the noninhibited reaction. By far the strongest inhibition was observed with TubB $\left(\mathrm{K}_{\mathrm{i}}=110 \mathrm{nM}\right)$, which was approximately 350-fold more effective than neomycin $B\left(K_{i}=38 \mu \mathrm{M}\right)$, one of the strongest $H H R$ inhibitors presently known. As a positive control, someknown H HR inhibitors ${ }^{12-14}$, such as neomycin $B$, gentamicin $C$, paramomycin, and neamine were tested, and their inhibitory potential was quantitatively confirmed in our assay. Furthermore, every inhibitor identified in the FRET assay was independently confirmed by classical polyacrylamide gel electrophoretic (PAGE) analysis using a radiolabeled substrate (see below).

We also screened filtrates from extracts of 2,000 different actinomycete strains with potential antibiotic activity. Each filtrate represented a fixed volume of cell-free supernatant passaged through an ultrafiltration membrane with a molecular-weight cutoff of $3,000 \mathrm{~g} / \mathrm{mol}$. The screening was performed as described for the 96

A

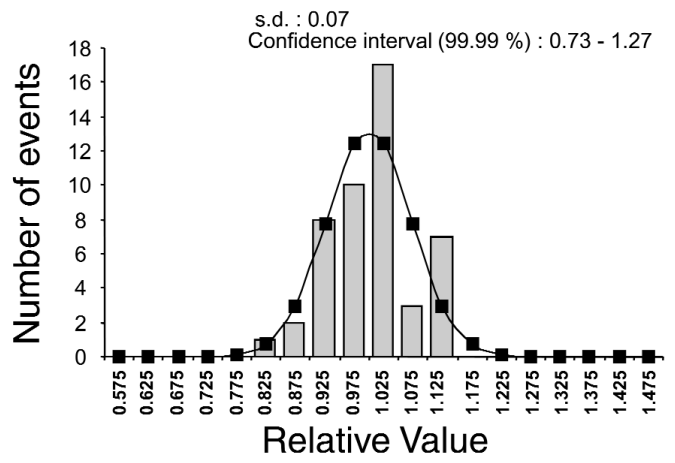

pure compounds, except that no concentration for the components could be calculated for the mixtures. In comparison to the noninhibited reaction, $\sim 5 \%$ of the extracts reduced the cleavage activity to a value of 0.7 or below under the conditions used (data not shown). This value corresponds to the inhibition obtained with $10 \mu \mathrm{M}$ neomycin, which was measured on the same plates as a positive control. From those extracts that showed inhibition, 58 samples were rescreened in triplicate measurements with almost the same results. M oreover, inhibition was confirmed independently by PAGE analysis (see below). Fractionation of the most active extracts and analyses of pure compound fractions are underway.

Several controls were performed to exclude the possibility that the inhibition obtained in the FRET-assay was due to unspecific fluorescence effects (Fig. 2A). In these control experiments a 5 '-32P-labeled substrate of the same sequence without FRET-labeling was used ${ }^{25}$. Reaction products were separated by denaturing PAGE, and product formation was quantified on a phosphorimager. No statistical differences between the FRET and PAGE analyses were obtained, thereby confirming all inhibitors by two independent analyses (Table 1). It should benoted, however, that for gramicidin S a significant deviation from the FRET data was obtained when we used the "classical" protocol for HHR-cleavage analysis, that is, removing samples for gel electrophoretic quantification at certain time points from a single reaction. For example, $100 \mu \mathrm{M}$ gramicidin $S$ exhibited a relative activity of 0.6 , whereas in the FRET assay the cleavage was almost completely inhibited. We anticipated that this inconsistency was due to aggregation of substrate and gramicidin $\mathrm{S}$.

B

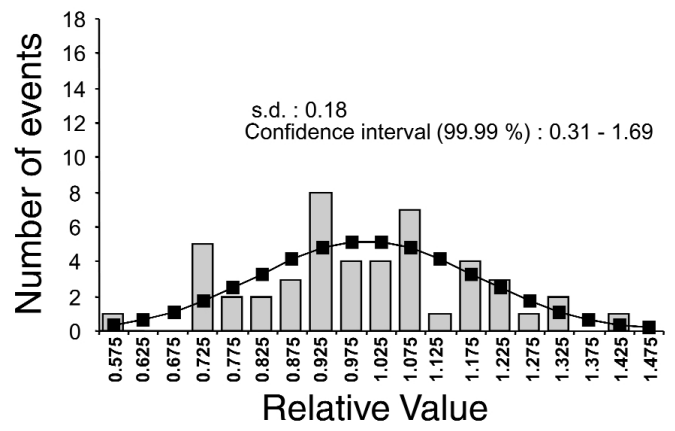

Figure 3. Statistical analysis of assay accuracy. Histogram plots showing the distributions of values obtained during a typical screening experiment (without any inhibitory compound) for $\mathbf{4 8}$ different measurements. The line represents a fitted Gaussian distribution, which was found to be adequate to approximate the distribution values for the relative activities $\left(r_{a}\right)$. (A) Histogram plots of the corrected slopes and (B) histogram plots of the corrected end points. The values are corrected for unspecific fluorescence (Experimental Protocol) and normalized to the average value of all measurements, that is, the mean value of the Gaussian function was set to 1 . The bars refer to the number of values obtained lying in the respective bin of size $\mathbf{0 . 0 5}$. Standard deviations as well as the 99.99 confidence intervals are also shown. 
A

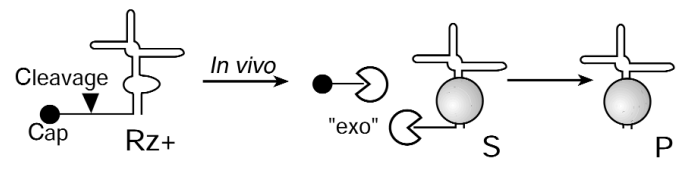

B

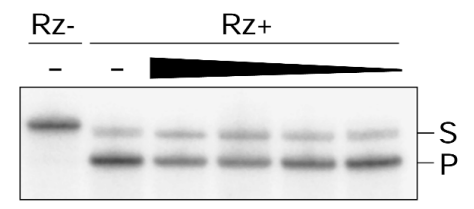

C

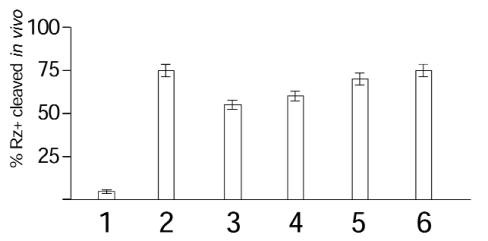

Figure 4. In vivo cleavage activity of the cis-snorbozyme in the presence and absence of TubA. (A) Schematic for the biochemical events leading to the final products in vivo. Uncleaved RNA is capped and thus protected from $5^{\prime} \rightarrow 3^{\prime}$ exonucleolytic degradation. Active snorbozyme is cleaved at the site indicated and then further trimmed by endogenous exonucleases as indicated, to yield the final in vivo product $P$, which is protected from complete degradation by proteins bound to the canonical snoRNA $C^{\prime} / D$ motif ${ }^{39}$. The assay makes it possible to distinguish true in vivo cleavage from artifactual cleavages in vitro, because the shorter product $P$ is formed only in living cells after ribozyme-dependent cleavage and not in vitro or after cell disruption. exo, Exonuclease activity. (B) Northern blot of one representative set of experiments. The same in-cis snorbozyme construct as described before with a hammerhead sequence (52 nucleotides) inserted into a full-size U3 variant was used ${ }^{37}$. RNA was identified by northern blot as described ${ }^{37}$ and quantified by phosphorimaging. Rz-, Inactive snorbozyme mutant negative control; $R z+$, active snorbozyme; $S$, fraction of $R z+$ that was not cleaved in vivo. $P, R z+$ cleaved in vivo. (C) Quantification of three independent measurements of snorbozyme cleavage in vivo. (1) Rz- (5 $\pm 3 \%)$; (2) Rz + uninhibited (75 \pm 5\%); (3) Rz+ plus $300 \mu \mathrm{M}$ TubA in the growth medium (55 $\pm 5 \%$ ); (4) $100 \mu$ M TubA (60 $\pm 5 \%$ ); (5) $30 \mu \mathrm{M}$ TubA (70 $\pm 5 \%)$; 6, $0 \mu \mathrm{M}$ TubA $(75 \pm 5 \%)$.

We therefore modified the protocol for the PAGE analysis by monitoring individual samples for each timepoint, which could be quantitatively analyzed by PAGE. Indeed, by employing this modification we obtained a relative activity of 0.03 , thereby establishing gramicidin $\mathrm{S}$ as a very potent inhibitor of hammerhead ribozyme cleavage (data not shown).

Kinetic characterization of new HHR inhibitors. For compounds exhibiting a strong effect on $H H R$ cleavage, $K_{i}$ values were determined by measuring the initial reaction velocities at increasing inhibitor concentrations. Representative results are shown in Figure 2B,C and Table 1. As control, a selection of six more or less inhibitory antibiotics were chosen and remeasured from independently diluted stock solutions ( Table 1, entries 8, 27, 40, 41, 53, 62). N one of the $\mathrm{K}_{\mathrm{i}}$ values obtained in this analysis deviated by $>20 \%$ from the values of the initial FRET measurements shown in Table 1. Interestingly, curve fitting revealed a positive cooperativity for the inhibition by TubA (Fig. 2C, cooperativity factor: 1.5).

Interestingly, some striking similarities and interesting differences become evident when inhibitors of other ribozymes ${ }^{27-30}$ are compared with our results. For example, among the aminoglycosides, 5-epi-sisomicin was identified as the strongest hammerhead inhibitor, with a $\mathrm{K}_{i}$ of $1 \mu \mathrm{M}$, followed by gentamicin C complex $(10 \mu \mathrm{M})$, neomycin sulfate $(18 \mu \mathrm{M})$, gentamicin C1A sulfate $(32 \mu \mathrm{M})$, neomycin B $(38 \mu \mathrm{M})$, paramomycin $(65 \mu \mathrm{M})$, and neamine $(90 \mu \mathrm{M})$. 5-epi-Sisomicin and gentamicin also showed strong inhibitory potential for the sunY intron and the HDV ribozyme ${ }^{21}$. Inhibition of the
HHR by neomycin is comparable to the HDV ribozyme; inhibition by paramomycin is comparable to the value of $\mathrm{K}_{i}$ obtained for the sun $\mathrm{Y}$ intron.

Whereas the cyclic pentapeptide antibiotics TubA and TubB showed very strong inhibition, other peptide antibiotics of the same family, namely TubN (enviomycin), viomycin, and capreomycin (capastat) were much less effective or had no effect at all. Interestingly, the strongest HHR inhibitors TubA and TubB both contain an $\mathrm{OH}$ group in $\alpha$-position to the guanidino group in the six-membered ring. All noninhibitory tuberactinomycins, except viomycin, lack this functionality. A similar trend is found for the sunY intron and the HDV ribozyme ${ }^{21}$, but here viomycin exhibits moderateinhibitory potential.

The striking difference in inhibitory activity found for viomycin and TubB in our study is remarkable because these two peptide antibiotics are considered to be identical. Our data strongly suggest that the two samples referred to as TubB and viomycin obviously differ in some respect and thus cannot be considered identical. Indeed, the two samples were isolated from different streptomycete strains, and have different counter-ions: "viomycin". $\mathrm{H}_{2} \mathrm{SO}_{4}$ from Streptomyces vinaceus; "TubB" $\cdot \mathrm{HCl}$ from Streptomyces griseoverticillus. At present, we do not know whether this may explain differences in the inhibitory potential of viomycin and TubB.

The third class of inhibitors identified in our screen belongs to the tetracycline family. Previously, tetracycline was established as a moderate HHR inhibitor ${ }^{12}$ and this was confirmed by our study. Chelocardin exhibited significantly stronger inhibitory potential. No inhibition was detected for oxytetracycline.

For the anthracycline antibiotic Adriamycin (doxorubicin), which belongs to the family of aromatic type II polyketides, a $K_{i}$ of $23 \mu \mathrm{M}$ was measured. D oxorubicin is known to interact with DNA and was recently shown to bind reversibly to RNA helices and to tRNA ${ }^{31}$.

A novel class of potent hammerhead inhibitors is represented by two dye molecules that have not yet been shown to affect ribozyme catalysis, namely bisbenzimide (Hoechst 33258), and the bis- $\mathrm{N}$-acetylglycine salt of 4,4'-(diazoamino) di-benzamidine (diminazene aceturate), which showed $\mathrm{K}_{i}$ values of $3 \mu \mathrm{M}$ and $20 \mu \mathrm{M}$, respectively. To our knowledge, bisbenzimidehas not been identified as an inhibitor of ribozyme activity but was recently shown to be a weak inhibitor $(>>100 \mu \mathrm{M})$ of the Tat/TAR interaction in vitro ${ }^{32,33}$. Bisbenzimide was also used as a target for an RNA aptamer selection $^{34}$, and the consensus motif 5'-U UAN ${ }_{4-5} \mathrm{UCU}-3^{\prime}$ was identified in the selected sequences. This motif is not present in HHR1. The second dyeinhibitor, diminazene aceturate, exhibits a $K_{i}$ of $20 \mu \mathrm{M}$. This drug was shown to interact with TAR RNA (ref. 35) and an RNA duplex ${ }^{36}$, probably by a combination of intercalation plus minorgroovebinding.

Statistical analysis. To ensure the assay's general applicability for the high-throughput screening of large compound libraries, we had to ensure that it can identify inhibitors reliably and accurately. We therefore statistically evaluated two different sets of data that may be utilized for the analysis of a screening experiment: the initial slopes of the fluorescence signal reflecting the cleavage kinetics that were used throughout this study, and the percentage of cleavage at a defined time point after the start of the reaction. To determine which of the two options would lead to highest accuracy, 48 measurements of the uninhibited reaction wereanalyzed. Figure $3 \mathrm{~A}$ shows the distribution of the corrected slopes (see Fig. 1C,D) fitted into a Gaussian distribution, from which confidence intervals were inferred. An analogous analysis for the corrected relative fluorescence values obtained after 4 min of measurement is shown in Figure 3B.

Thestandard deviations for the two analyses show significant differences. For the analysis based on the initial slopes the standard deviation was $7 \%$, compared to $18 \%$ for the single-point analysis. The confidence interval, that is, the value one would expect for 
99.99\% of all experiments, ranged between 0.73 and 1.27 for the slope-based data. Therefore, the risk of obtaining false positives is one in $10^{4}$ if the threshold for inhibition is set to 0.7 relative to the uninhibited reaction. For the single-point analysis the confidence interval ranged between 0.31 and 1.69. This does not mean that the analysis based on single- point determinations is not useful, especially in cases in which a lower threshold (0.3) is sufficient. For a rapid analysis of large libraries of individual compounds it might be advantageous to choose single point analysis because it may, for example, allow the screening of a larger number of compounds in a primary screen because of the shorter time intervals required. In conclusion, the statistical evaluation shows that our assay is capable of detecting even very weak inhibitory activities. This is less relevant if individual, defined compounds are screened but may become highly relevant if more or less complex mixtures are searched in a primary screen, as in the case of screening 2,000 different bacterial extracts of mixed composition.

Cleavage of a H HR snorbozyme is reduced by TubA in vivo. To investigate whether some of the identified H HR inhibitors can also act inside cells, we employed the "snorbozyme" methodology, which utilizes U3 small nucleolar RNA-hammerhead ribozyme hybrids to accurately and reliably assay ribozyme activity in vivo $^{37,38}$. Using the cis-snorbozyme (Fig. 4A), we evaluated the intracellular inhibition of H H R cleavage by six inhibitors, namely bisbenzimide, TubA, TubB, doxorubicin, diminazene aceturate, and 5 -epi-sisomicin in yeast cells. Cells were grown at various permissive concentrations of inhibitors (TubB and bisbenzimide exhibited cell toxicity at $100 \mu \mathrm{M}$ and $10 \mu \mathrm{M}$ concentrations, respectively; other compounds were toxic at $1.0 \mathrm{mM}$ ), the RNA was isolated at the same growth stages, and snorbozyme substrates and cleavage products were subsequently quantified by northern blotting. TubA was the only compound for which a reduction of H H R cleavage in vivo was observed (Fig. 4B,C; data for the remaining inactive compounds not shown). TubA added to the growth medium at $300 \mu \mathrm{M}$ reduced snorbozyme cleavage in vivo by nearly $30 \%$ (compare lanes 2 and 3). Although not dramatic, the effect was clearly concentration-dependent and highly reproducible within three independent experiments. To our knowledge, this is the first example showing that ribozyme-activity can be reduced by a small molecule in vivo.

It is not completely clear why some compounds that are potent inhibitors in vitro do not show activity in vivo. For TubB and bisbenzimine, the explanation can be as simple as that their high toxicity does not allow them to reach the intracellular concentration of the compound necessary for inhibition. For others, such reasons as low permeability of cell membranes and low accessibility of the ribozyme segment as part of the RNP complex might provide an explanation. With ribozymes as in vivo targets, factors such as the mechanism of inhibition may also be important. In this context it is interesting to note that TubA, the only compound active in vivo, showed positive cooperativity of HHR inhibition in vitro with a cooperativity coefficient of 1.5 (Fig. 2). This effect was not observed with TubB, which points to slight differences in theinhibitory mechanisms that may play some rolein vivo.

In summary, wehave described a new approach for the rapid and convenient identification of new ribozyme inhibitors from a small, constrained antibiotic library. With a nonautomated, nonoptimized laboratory prototype we could easily analyze at least 480 different ribozyme reactions per day. This approach should be generally applicablefor the rapid identification of low-molecular-weight modulators of functional RNAs. These are lead candidates not only for facilitating our understanding of RNA-drug interaction but also for providing valuable information for the rational design of new antibiotic reagents targeting RNA. The approach can al so serve as a starting point for the development of high-throughput screening systems directed against a variety of RNA-catalyzed or RNA-modulated cellular processes such as bacterial mRNA splicing. Toward this end, we showed that inhibition of hammerhead ribozyme catalysis was reliably detectable within 2,000 uncharacterized bacterial extracts. In general, low-molecular-weight molecules that bind to RNA have the potential to be used as drugs, which would open up a whole new line of investigation for the pharmaceutical industry.

\section{Experimental protocol}

Kinetic measurements. Kinetic parameters of ribozyme cleavage were determined under multiple-turnover conditions with $8 \mathrm{nM} \mathrm{HHR1}$ and $200 \mathrm{nM}$ FRET-substrate SL1 using a Biolumin 960 fluorescence reader (M olecular Dynamics, Sunnyvale, CA). All reactions were carried out in $50 \mu \mathrm{l}$ final volume in $0.5 \times \mathrm{PBS}$ with $8.0 \mathrm{mM} \mathrm{M} \mathrm{gCl}{ }_{2}$ at $32^{\circ} \mathrm{C}$ and $\mathrm{pH} 7.4$. Preceding each measurement, stock solutions of ribozyme and substrate were incubated separately at $85^{\circ} \mathrm{C}$ (H HR1 for $3.5 \mathrm{~min}$ in $1 \times$ PBS; SL 1 for $1.5 \mathrm{~min}$ in water), cooled to $32^{\circ} \mathrm{C}$ within $15 \mathrm{~min}$, combined and, after $15 \mathrm{~min}$, dispensed on 96-well plates $\left(30 \mu \mathrm{l} /\right.$ well). Reactions were initiated by adding $\mathrm{M} \mathrm{gCl}_{2}$ and antibiotic simultaneously. Fluorescence was recorded at 14 sintervals. Raw data were exported to M icrosoft Excel and analyzed with a program that is available on request. Negative control reactions with an inactive HHR1 mutant ${ }^{25}$ or without H HR1 were recorded in parallel. Initial reaction velocities were determined by linear fitting. All rates ( $v$ in Fu/min) were corrected for unspecific fluorescence (see above) by subtracting the slopes of the control sample $\left(v_{c}\right)$ from the corresponding slopes obtained in the hammerhead reaction $\left(v_{H H R}\right)$. Analogously, values obtained for the single-point measurements of our statistical analysis were corrected by subtraction of the intrinsic fluorescence of uncleaved substrate measured in the control sample without HHR1.

Initial slopes in the presence of $100 \mu \mathrm{M}$ antibiotic. For a typical screening experiment, master mixes for 240 cleavage- and control reactions without ribozyme were prepared. Eight different compounds plus controls and noninhibited reactions as internal standards were assayed in triplicates per plate. Initial rates $\left(\mathrm{s}_{\mathrm{f}}\right.$ ) were calculated as the slope of the linear regression fitting the increase of fluorescence in the reaction well and were corrected by subtraction of the control value $\left(s_{c}\right)$ without HHR1. The corrected initial rate $(v)$ is thus $v=s_{r}-s_{c}$. The mean value of 48 measurements in the absence of inhibitors was $\left(v_{\text {ref }}\right)$. Finally, the corrected initial rates were normalized relatively to $v_{\text {ref, }}$ giving the relative ribozyme activity $\left(r_{a}=v / v_{\text {ref }}\right)$. Analyses with ${ }^{32} \mathrm{P}$-labeled substrate $\mathrm{S} 1$ were done analogously using the standard protocols based on gel electrophoretic separation and phosphorimager quantification.

Inhibition constants $\left(\mathbf{K}_{\mathbf{i}}\right) . \mathrm{K}_{\mathrm{i}}$ values for antibiotic inhibition of ribozyme cleavage were obtained by measuring the initial reaction velocities at different antibiotic concentrations. For each experiment, a 240× mix of SL1 with and without HHR1 was prepared and transferred into six plates ( $30 \mu \mathrm{l} /$ well). Each plate contained four different antibiotics at eight different concentrations, measured in triplicates. For analysis, the relative activity ( $r_{a}$; see above) was plotted against the inhibitor concentration (c), and $\mathrm{K}_{\mathrm{i}}$ values were determined by fitting the data points to the equation $\left(1+c / K_{i}\right)^{-1}$ using the least square method (TubA was fitted to $\left[1+\left(c / K_{i}\right)^{n}\right]^{-1}$, wheren is a cooperativity factor).

In vivo inhibition studies. Yeast cells expressing active cis-snorbozyme (plasmid pRS:T/C(+); see ref. 37) were grown overnight in $5 \mathrm{ml}$ of YNB $(0.67 \%$ yeast nitrogen base) selective medium, containing $2 \%$ glucose and indicated amounts of inhibitors. Cells expressing inactive (mutant) cis-snorbozyme (pRS:T/C(-); see ref. 37) were grown in the same medium without inhibitors. In cases where inhibitors turned out to be toxic, reduced cell growth was observed. The RNA was isolated from the surviving cells at approximately the same growth stage $\left(0.8-1.0 O_{600}\right)$, and subjected to northern blotting with the 32P-labeled oligonucleotide SD 13* (5'-GCGGCTTAGGCTAAGCTAAGGCCAGC-3'), specific to the snorbozyme molecule ${ }^{37}$. Quantification was by phosphorimaging.

\section{Acknowledgments}

We thank Barbara Waters and TerraGen Discovery for providing the actinomycete extracts and Abbott Laboratories for chelocardin, E. Bertrand, E. Westhof, and the members of the Famulok laboratory for helpful discussions, and $M . J$. Fournier for sharing the strategic approach of the in vivo cleavage assay. This work was supported by the Deutsche Forschungsgemeinschaft, Aventis GenCell (to M.F. and N.P.), and the H. Arthur Smith Foundation for Cancer Research (to D.A.S.). 
1. Gait, M.J . \& Karn, J. RNA recognition by the human immunodeficiency virus Tat and Rev proteins. Trends Biochem. Sci. 18, 255-259 (1993).

2. Hermann, T. \& Westhof, E. RNA as a drug target: chemical, modelling, and evolutionary tools. Curr. Opin. Biotechnol. 9, 66-73 (1998).

3. Afshar, M., Prescott, C.D. \& Varani, G. Structure-based and combinatorial search for new RNA-binding drugs. Curr. Opin. Biotechnol. 10, 59-63 (1999).

4. Ecker, D.J . \& Griffey, R.H. RNA as a small-molecule drug target: doubling the value of genomics. Drug Discovery Today 4, 420-429 (1999).

5. Woodcock, J ., Moazed, D., Cannon, M., Davies, J. \& Noller, H.F. Interaction of antibiotics with A- and P-site-specific bases in $16 \mathrm{~S}$ ribosomal RNA. EMBO J . 10, 3099-3103 (1991).

6. Zapp, M.L., Stern, S. \& Green, M.R. Small molecules that selectively block RNA binding of HIV-1 Rev protein inhibit Rev function and viral production. Cell 74, 969-978 (1993).

7. Wang, S., Huber, P.W., Cui, M., Czarnik, A.W. \& Mei, H.Y. Binding of neomycin to the TAR element of HIV-1 RNA induces dissociation of Tat protein by an allosteric mechanism. Biochemistry 37, 5549-5557 (1998)

8. Wang, Y., Hamasaki, K. \& Rando, R.R. Specificity of aminoglycoside binding to RNA constructs derived from the 16S rRNA decoding region and the HIV-RRE activator region. Biochemistry 36, 768-779 (1997).

9. Walter, F., Vicens, Q. \& Westhof, E. Aminoglycoside-RNA interactions. Curr. Opin. Chem. Biol. 3, 694-704 (1999).

10. Schroeder, R., Waldsich, C. \& Wank, H. Modulation of RNA function by aminoglycoside antibiotics. EMBO J . 19, 1-9 (2000).

11. Hermann, T. \& Westhof, E. Aminoglycoside binding to the hammerhead ribozyme: a general model for the interaction of cationic antibiotics with RNA. J . Mol. Biol. 276, 903-912 (1998).

12. Murray, J.B. \& Arnold, J.R. Antibiotic interactions with the hammerhead ribozyme: tetracyclines as a new class of hammerhead inhibitor. Biochem. J. 317, 855-860 (1996)

13. Clouet-d'Orval, B., Stage, T.K. \& Uhlenbeck, O.C. Neomycin inhibition of the hammerhead ribozyme involves ionic interactions. Biochemistry $\mathbf{3 4}$ 11186-11190 (1995).

14. Stage, T.K., Hertel, K.J. \& Uhlenbeck, O.C. Inhibition of the hammerhead ribozyme by neomycin. RNA 1, 95-101 (1995).

15. Michael, K. \& Tor, Y. Designing novel RNA Binders. Chemistry Eur. J. 4, 2091-2098 (1998).

16. Scott, W.G., Murray, J B., Arnold, J R.P., Stoddard, B.L. \& Klug, A. Capturing the structure of a catalytic RNA intermediate: the hammerhead ribozyme. Science 274, 2065-2069 (1996)

17. Pearson, N.D. \& Prescott, C.D. RNA as a drug target. Chem. Biol. 4, 409-414 (1997).

18. Stage-Zimmermann, T.K. \& Uhlenbeck, O.C. Hammerhead ribozyme kinetics. RNA 4, 875-889 (1998)

19. Tor, Y. RNA and the small molecule world. Angew. Chem. Int. Edn. 38, 1579-1582 (1999).
20. Noller, H.F. Ribosomes. Drugs and the RNA world. Nature 353, 302-303 (1991).

21. Rogers, J ., Chang, A.H., von Ahsen, U., Schroeder, R. \& Davies, J . Inhibition of the self-cleavage reaction of the human hepatitis delta virus ribozyme by antibiotics. J. Mol. Biol. 259, 916-925 (1996).

22. Neu, H.C. The crisis in antibiotic resistance. Science 257, 1064-1073 (1992)

23. Davies, J. \& Wright, G.D. Bacterial resistance to aminoglycoside antibiotics. Trends Microbiol. 5, 234-240 (1997).

24. Davies, J Inactivation of antibiotics and the dissemination of resistance genes Science 264, 375-382 (1994).

25. J enne, A., Gmelin, W., Raffler, N. \& Famulok, M. Real-time characterization of ribozymes by fluorecence resonance energy transfer (FRET). Angew. Chem. Int. Edn. 38, 1300-1303 (1999).

26. Singh, K.K., Parwaresch, R. \& Krupp, G. Rapid kinetic characterization of hammerhead ribozymes by real-time monitoring of fluorescence resonance energy transfer (FRET). RNA 5, 1348-1356 (1999).

27. von Ahsen, U., Davies, J. \& Schroeder, R. Non-competitive inhibition of group intron RNA self-splicing by aminoglycoside antibiotics. J. Mol. Biol. 226 935-941 (1992)

28. Hoch, I., Berens, C., Westhof, E. \& Schroeder, R. Antibiotic inhibition of RNA catalysis: neomycin $B$ binds to the catalytic core of the td group I intron displacing essential metal ions. J. Mol. Biol. 282, 557-569 (1998).

29. Mikkelsen, N.E., Brannvall, M., Virtanen, A. \& Kirsebom, L.A. Inhibition of RNase $P$ RNA cleavage by aminoglycosides. Proc. Natl. Acad Sci. USA 96, 6155-6160 (1999).

30. Earnshaw, D.J . \& Gait, M.J . Hairpin ribozyme cleavage catalyzed by aminoglycoside antibiotics and the polyamine spermine in the absence of metal ions. Nucleic Acids Res. 26, 5551-5561 (1998).

31. Zhu, K., Henning, D., Iwakuma, T., Valdez, B.C. \& Busch, H. Adriamycin inhibits human RH II/Gu RNA helicase activity by binding to its substrate. Biochem. Biophys. Res. Commun. 266, 361-365 (1999).

32. Dassonneville, L., Hamy, F., Colson, P., Houssier, C. \& Bailly, C. Binding of Hoechst 33258 to the TAR RNA of HIV-1. Recognition of a pyrimidine bulgedependent structure. Nucleic Acids Res. 25, 4487-4492 (1997).

33. Mestre, $B$. et al. Oligonucleotide inhibition of the interaction of HIV-1 Tat protein with the trans-activation responsive region (TAR) of HIV RNA. Biochim. Biophys. Acta 1445, 86-98 (1999).

34. Werstuck, G. \& Green, M.R. Controlling gene expression in living cells through small molecule-RNA interactions. Science 282, 296-298 (1998).

35. Bailly, C.. Colson, P., Houssier, C. \& Hamy, F. The binding mode of drugs to the TAR RNA of HIV-1 studied by electric linear dichroism. Nucleic Acids Res. 24 1460-1464 (1996).

36. Pilch, D.S., Kirolos, M.A., Liu, X., Plum, G.E. \& Breslauer, K.] . Berenil [1,3-bis (4' amidinophenyl)triazene] binding to DNA duplexes and to a RNA duplex: evidence for both intercalative and minor groove binding properties. Biochemistry 34, 9962-9976 (1995)

37. Samarsky, D.A. et al. A small nucleolar RNA:ribozyme hybrid cleaves a nucleolar RNA target in vivo with near-perfect efficiency. Proc. Natl. Acad. Sci. USA 96, 6609-6614 (1999).

38. Rossi, J J. Ribozymes in the nucleolus. Science 285, 1685 (1999).

39. Samarsky, D.A., Fournier, M.J., Singer, R.H. \& Bertrand, E. The snoRNA box C/D motif directs nucleolar targeting and also couples snoRNA synthesis and localization. EMBO J . 17, 3747-3757 (1998) 